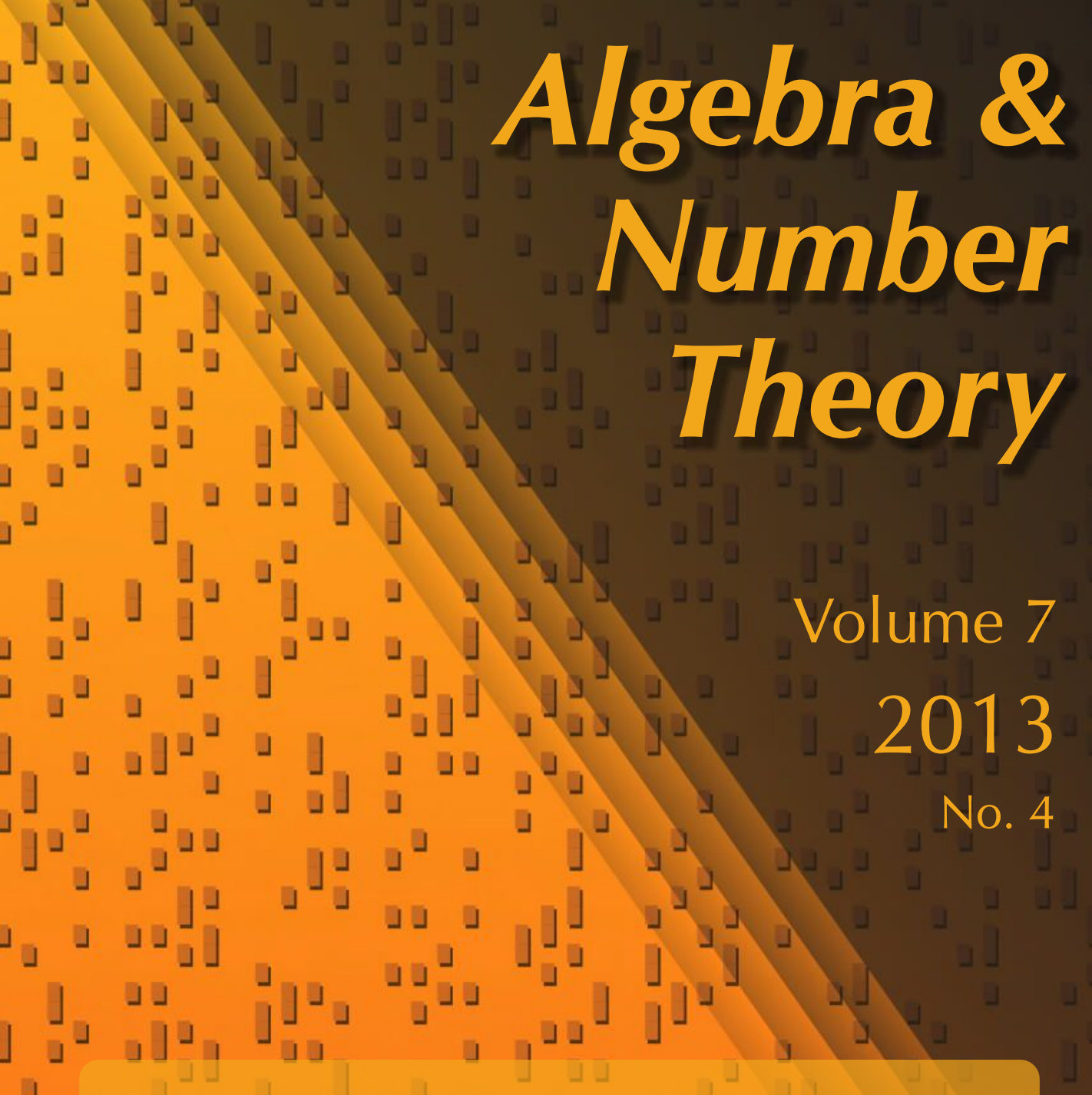




\title{
An analogue of Sturm's theorem for Hilbert modular forms
}

\author{
Yuuki Takai
}

\begin{abstract}
In this paper, we consider congruences of Hilbert modular forms. Sturm showed that $\bmod \ell$ elliptic modular forms of weight $k$ and level $\Gamma_{1}(N)$ are determined by the first $(k / 12)\left[\Gamma_{1}(1): \Gamma_{1}(N)\right] \bmod \ell$ Fourier coefficients. We prove an analogue of Sturm's result for Hilbert modular forms associated to totally real number fields. The proof uses the positivity of ample line bundles on toroidal compactifications of Hilbert modular varieties.
\end{abstract}

\section{Introduction}

In this paper, we consider congruences of Hilbert modular forms. Sturm [1987, Theorem 1] showed that mod $\ell$ modular forms of weight $k$ and level $\Gamma_{1}(N)$ are determined by the first $(k / 12)\left[\Gamma_{1}(1): \Gamma_{1}(N)\right] \bmod \ell$ Fourier coefficients. We prove an analogue of Sturm's result for Hilbert modular forms associated to totally real number fields not equal to $\mathbb{Q}$.

Doi and Ohta [1977, Lemma 2.1] showed a result similar to Sturm's theorem for elliptic cusp forms of weight 2 by a geometric method. Sturm improves the result for general weights and general levels by a technical method. For the case that the coefficient field is $\mathbb{C}$, the similar result was long known [Miyake 1989, Corollary 2.3.4]. Recently, Baba, Chakraborty and Petridis [Baba et al. 2002, Theorem 3] obtained its generalization for complex Hilbert modular forms by using the Rayleigh quotient for the Laplace operator. It seems difficult to apply their method to the mod $\ell$ case. For the $\bmod \ell$ case, Burgos Gil and Pacetti [Dieulefait et al. 2010, Appendix B] showed a generalization for Hilbert modular forms associated to $\mathbb{Q}(\sqrt{5})$ and level $\Gamma_{0}(6 \sqrt{5})$ by a method similar to ours.

As mentioned above, the aim of the article is to prove an analogue of Sturm's theorem for Hilbert modular forms. In other words, we obtain an upper bound of

Supported by MEXT Grant-in-Aid for Young Scientists (B) 23740011, JSPS Grant-in-Aid for Scientific Research (B) 21340004, and JSPS Grant-in-Aid for Young Scientists (S) 21674001.

MSC2010: primary 11F41; secondary 11F30, 11F33, 14C17.

Keywords: Hilbert modular forms and varieties, congruences of modular forms, Sturm's theorem, toroidal and minimal compactifications, intersection numbers. 
the order at zeros of Hilbert modular forms at the exceptional locus of resolution of cusp singularities.

To explain our main result, we prepare several notions (see Sections 2.1-2.2 for more precise definitions). Let $N \geq 3$ be an integer, $F$ a totally real number field of finite degree $g \geq 2, O_{F}$ the ring of integers of $F, d_{F}$ the discriminant of $F$, and $\mathfrak{c}$ a nonzero integral ideal of $F$. Let $\widetilde{F}$ be the Galois closure of $F$ and $O_{\widetilde{F}}$ the ring of integers of $\widetilde{F}$. For a field $K$ that is an $\widetilde{O}_{\widetilde{F}}\left[1 /\left(N d_{F}\right), \mu_{N}\right]$-algebra, $M_{K}=M_{K}(\mathfrak{c}, N)$ denotes the open connected Hilbert modular variety over $K$ defined as a moduli of $\mathfrak{c}$-polarized Hilbert-Blumenthal abelian varieties with $\Gamma(N)$-structure. Then $\bar{M}_{K}=\bar{M}_{K, \Sigma}$ denotes the toroidal compactification associated to a collection $\Sigma$ of cone decompositions, $\underline{\omega}^{k}=\otimes_{i} \underline{\omega}_{i}^{k_{i}}$ denotes the automorphic line bundle on $\bar{M}_{K}$ of weight $k=\left(k_{1}, \ldots, k_{g}\right) \in \mathbb{Z}_{\geq 0}^{g}$, and $M^{*}$ denotes the minimal compactification of $M$. When $k=(1,1, \ldots, 1)$, we write $\underline{\omega}$ instead of $\underline{\omega}^{k}$. Our main result is the following:

Theorem 1. Let $N \geq 3$ be an integer, $k=\left(k_{1}, k_{2}, \ldots, k_{g}\right) \in \mathbb{Z}_{\geq 0}^{g}, \mathfrak{c}$ a nonzero integral ideal of $F, K$ a field that is an $\widetilde{O}_{\widetilde{F}}\left[1 /\left(N d_{F}\right), \mu_{N}\right]$-algebra $\left(a \mathbb{Z}\left[1 /\left(N d_{F}\right), \mu_{N}\right]\right.$ algebra if $k$ is parallel), and $S$ a nonempty finite set of irreducible components of codimension 1 in $\bar{M}_{K} \backslash M_{K}$. Let $f$ be a c-polarized geometric Hilbert modular form over $K$ of weight $k$ and level $\Gamma(N)$, i.e., $f \in H^{0}\left(M_{K}(\mathfrak{c}, N), \underline{\omega}^{k}\right)$. Then

$$
f \not \equiv 0 \Rightarrow \min _{E \in S}\left\{\operatorname{ord}_{E}(f)\right\}<\kappa
$$

for

$$
\kappa=\kappa_{S}=\kappa_{S}(k, N)=C^{g-1} \sum_{i=1}^{g} \frac{k_{i}\left\{\left(\underline{\omega}^{(g-1)} \cdot \underline{\omega}_{i}\right)+\left(\mathfrak{I}^{(g-1)} \cdot \underline{\omega}_{i}\right)\right\}}{\left(\mathfrak{I}^{(g-1)} \cdot \sum_{E \in S} E\right)},
$$

where $C$ is a positive integer independent of $k$ and $N, \mathfrak{I}$ is the inverse image of the ideal sheaf defining $M_{K}^{*} \backslash M_{K}$ by $\pi: \bar{M}_{K} \rightarrow M_{K}^{*}$, and the dot $(\cdot)$ denotes the intersection number (see Section 2.1).

The reason why we call Theorem 1 an "analogue" of Sturm's theorem is that the constant $\kappa$ includes a strange constant $C$. When $g=2$ and the canonical divisor $K_{\bar{M}}$ of $\bar{M}$ is nef (numerically effective), we may take an explicit constant as $\kappa$ (Theorem 16). Moreover, when the field $K$ is of positive characteristic and $\bar{M}$ is a minimal surface of general type, using Ekedahl's result [1988, Chapter III, Proposition 1.13], we may take a slightly better form of $\kappa$ than Theorem 16 (Theorem 19). Applying Theorem 1 to classical Hilbert modular forms, we obtain the more useful results for the complex case (Corollary 9) and the $\bmod \ell$ case (Corollary 12). Corollary 9 gives another proof of [Baba et al. 2002, Theorem 3]. As another application of Theorem 1, we obtain a rough upper bound of the dimension of vector space of Hilbert modular forms (Corollary 15). Because the dimension for weight 1 is unknown, Corollary 15 is not trivial. We remark that 
we can also show the results for the congruence subgroup $\Gamma_{1}$ by changing $\Gamma$ in the proofs to $\Gamma_{1}$ and using theory of the arithmetic compactifications of Hilbert modular varieties for $\Gamma_{1}$ by Dimitrov [2004].

Theorem 1 is proved by a method extending Doi and Ohta's algebraic geometric one. To obtain an upper bound of the order of zeros of modular forms at cusps, Doi and Ohta used Riemann-Roch's theorem on modular curves over finite fields. However, to obtain the bound for Hilbert modular forms, we use the positivity of ample line bundles. The key point of the proof is the construction of a specific ample line bundle on the toroidal compactification. To do this, we use the semiampleness of the automorphic line bundle on the minimal compactification proved by MoretBailly [1985, Chapter V, Theorem 2.1]. Combining the inverse image of the ample line bundle onto a toroidal compactification with a certain relatively ample line bundle, a specific ample line bundle on the toroidal compactification is constructed.

This article is organized as follows. In Section 2.1, the notation of intersection numbers and the two facts of ampleness are explained. In Section 2.2, the definitions and the properties of integral models of Hilbert modular varieties, these arithmetic toroidal and minimal compactifications, and the geometric Hilbert modular forms are recalled. In Section 3.1, for Hilbert modular forms associated to totally real number fields that are not $\mathbb{Q}$, Theorem 1 is proved. As consequences of Theorem 1 , Corollaries 9-15 are obtained. In Section 3.2, for the case that $F$ is a real quadratic field, we obtain more explicit forms for $\kappa$ in Theorems 16 and 19.

\section{Preliminaries}

\section{Notation and conventions.}

- Let $F$ denote a totally real number field and $g=[F: \mathbb{Q}]<\infty$. Let $\mathfrak{O}_{F}$, $\mathfrak{d}=\mathfrak{d}_{F}$, and $d_{F}$ be the ring of integers of $F$, the different ideal of $F / \mathbb{Q}$, and the discriminant of $F / \mathbb{Q}$, respectively. Furthermore, $I=I_{F}$ denotes the set of the embeddings of $F$ into $\mathbb{R}$, and $F_{\infty}=F \otimes_{\mathbb{Q}} \mathbb{R}$.

- For a nonzero fractional ideal $\mathfrak{a}$ of $F, \mathfrak{a}^{*}=\mathfrak{a}^{-1} \mathfrak{d}^{-1}$, and $(\mathfrak{a})_{+}$denotes the subset of $\mathfrak{a}$ consisting of the totally positive elements.

- Let $\mathbf{S c h}_{/ R}$ denote the category of the schemes over a ring $R, R$-Alg the category of the $R$-algebras, and Sets the category of the sets.

- Let $X$ be a normal variety. For a Cartier divisor $D$ on $X,[D]$ denotes the Weil divisor associated to $D$. For a rational function $f$ on $X$, $\operatorname{div}(f)$ denotes the divisor associated to $f$. A $\mathbb{Q}$-Cartier divisor $D$ on $X$ is a divisor such that $m D$ is a Cartier divisor for a nonzero integer $m$.

- For two functions $f, g: \mathbb{R}^{n} \rightarrow \mathbb{R}_{\geq 0}, f \ll g$ denotes that there is a positive constant $A$ such that $f(x) \leq A g(x)$ for all $x \in \mathbb{R}^{n}$. 
2.1. Intersection numbers and ampleness. In this section, we recall some facts in intersection theory [Fulton 1998, Chapter 2; Lazarsfeld 2004, Chapter 1].

Let $d$ and $n$ be positive integers such that $d<n, K$ a field, and $X$ a normal proper variety of dimension $n$ over $K$. For Cartier divisors $D_{1}, D_{2}, \ldots, D_{d}$ on $X$ and a $d$-dimensional irreducible closed subvariety $V$ of $X$, the intersection number of $D_{1}, D_{2}, \ldots, D_{d}$ and $V$, denoted $\left(D_{1} \cdots D_{d} \cdot V\right)$, is defined by several methods. But it is unique [Hartshorne 1977, Appendix A]. If $D=D_{1}=\cdots=D_{d}$, then we write $\left(D^{(d)} \cdot V\right)$ instead of $\left(D_{1} \cdots D_{d} \cdot V\right)$. For a Cartier divisor $E$, the intersection number $\left(D_{1} \cdots D_{n-1} \cdot E\right)$ of $D_{1}, \ldots, D_{n-1}$ and $E$ is defined by $\left(D_{1} \cdots D_{n-1} \cdot[E]\right)$ and linearity. If $D=E=D_{1}=\cdots=D_{n-1}$, we write $\left(D^{(n)}\right)$ instead of $\left(D^{(n-1)} \cdot D\right)$. For line bundles $\mathscr{L}_{i} \simeq \mathfrak{O}_{X}\left(D_{i}\right)$ for $1 \leq i \leq n-1$ and $\mathscr{L}^{\prime} \simeq \mathfrak{O}_{X}(E)$, the intersection number $\left(\mathscr{L}_{1} \cdots \mathscr{L}_{n-1} \cdot \mathscr{L}^{\prime}\right)$ is defined as $\left(D_{1} \cdots D_{n-1} \cdot E\right)$. For $\mathbb{Q}$-Cartier divisors $D_{1}, \ldots, D_{n-1}$ and a $\mathbb{Q}$-Cartier divisor $E$, their intersection number $\left(D_{1} \cdots D_{n-1} \cdot E\right)$ is defined by $\left(m_{1} D_{1} \cdots m_{n-1} D_{n-1} \cdot m_{n} E\right) / m_{1} m_{2} \cdots m_{n}$, where $m_{i}$ are integers such that $m_{i} D_{i}$ and $m_{n} E$ are Cartier.

We recall a fact on the ampleness of line bundles:

Lemma 2 [Lazarsfeld 2004, Proposition 1.7.10]. Let $X$ and $Y$ be proper varieties, $f: X \rightarrow Y$ a proper morphism, $\mathscr{L}$ an $f$-ample line bundle on $X$, and $\mathcal{M}$ an ample line bundle on $Y$. Then the line bundle $\left(f^{*} \mathcal{M}\right)^{\otimes m} \otimes \mathscr{L}$ is ample on $X$ for a sufficiently large positive integer $m$.

We also recall that ampleness of line bundles is preserved by the pullback of finite morphisms.

Lemma 3 [Lazarsfeld 2004, Proposition 1.2.13, Corollary 1.2.28]. Let $X$ and $Y$ be two projective varieties, and let $f: X \rightarrow Y$ be a finite morphism. If a line bundle $\mathscr{L}$ on $Y$ is ample, then $f^{*} \mathscr{L}$ is ample. Moreover, when $f$ is finite and surjective, a line bundle $\mathscr{L}$ on $Y$ is ample if and only if $f^{*} \mathscr{L}$ is ample.

2.2. Hilbert modular varieties and geometric modular forms. Let $\mathfrak{c} \subset F$ be a fixed nonzero integral ideal, $N$ a positive integer, and $R$ a $\mathbb{Z}\left[1 /\left(N d_{F}\right)\right]$-algebra. For an $R$-algebra $B$, let $\underline{A}=\left(A, \iota, \lambda, \phi_{N}\right)$ be a c-polarized Hilbert-Blumenthal abelian variety with $\Gamma(N)$-structure over $B$; i.e.,

(1) $\rho: A \rightarrow \operatorname{Spec}(B)$ is an abelian scheme,

(2) $\iota: \mathbb{O}_{F} \hookrightarrow \operatorname{End}_{B}(A)$ is an injective ring homomorphism taking 1 to the identity,

(3) $\lambda$ is a c-polarization (see [Hida 2004, Section 4.1.1] for the definition),

(4) $\phi_{N}: A[N] \simeq\left(\mathbb{O}_{F} / N \mathscr{O}_{F}\right)^{2}$ is a $\Gamma(N)$-structure (i.e., an isomorphism as $\mathscr{O}_{F}$ modules), and

(5) $\rho_{*} \Omega_{A / \operatorname{Spec} B}^{1}$ is a locally free $O_{F} \otimes_{\mathbb{Z}} B$-module of rank 1 . 
A suitable rule defines the isomorphism on such schemes [Hida 2004, Section 4.1.1]. We consider the contravariant functor $\mathscr{E}=\mathscr{E}(\mathfrak{c}, \Gamma(N)): \mathbf{S c h}_{/ R}^{\mathrm{op}} \rightarrow$ Sets defined by

$$
\mathscr{E}(\mathfrak{c}, \Gamma(N))(B)=\left\{\left(A, \iota, \lambda, \phi_{N}\right)_{/ B} / \text { isom. }\right\} .
$$

The functor $\mathscr{E}(\mathfrak{c}, \Gamma(N))$ admits a coarse moduli scheme over $R$. When $N \geq 3$, $\mathscr{E}(\mathfrak{c}, \Gamma(N))$ is representable by a scheme over $R$. Its fine moduli scheme is denoted $M=M_{R}=M_{N}=M_{R, N}=M(\mathfrak{c}, \Gamma(N))=M_{R}(\mathfrak{c}, \Gamma(N))$, and the universal object is denoted $A^{U} \rightarrow M_{N}$. Remark that $M$ is naturally defined over $R\left[\mu_{N}\right]=R[x] /\left(\Phi_{N}(x)\right)$ [Deligne and Rapoport 1973, Section 3.20], where $\Phi_{N}$ is the $N$ th cyclotomic polynomial. Furthermore, $M$ is a smooth, geometrically irreducible scheme of finite type and relative dimension $g$ over $R\left[\mu_{N}\right]$.

Next we define the cusps of $M_{N}$. Let $\mathfrak{a}$ and $\mathfrak{b}$ be nonzero fractional ideals of $F$ such that $\mathfrak{c}=\mathfrak{a b}^{-1}$, and let

$$
\phi_{N}: \mathfrak{b} \oplus \mathfrak{a}^{*} / N\left(\mathfrak{b} \oplus \mathfrak{a}^{*}\right) \simeq\left(\mathscr{O}_{F} / N \mathscr{O}_{F}\right)^{2}
$$

be an isomorphism as $\mathrm{O}_{F}$-modules. We set

$$
\Gamma(N ; \mathfrak{a}, \mathfrak{b})=\left\{\left(\begin{array}{ll}
a & b \\
c & d
\end{array}\right) \in S L_{2}(F) \mid \begin{array}{c}
a, d \in 1+N \mathscr{O}_{F} \\
b \in N(\mathfrak{a} \mathfrak{b})^{*}, c \in N\left(\mathfrak{a} \mathfrak{b} \mathfrak{d}_{F}\right)
\end{array}\right\} .
$$

We define the action of $\gamma \in \Gamma(N ; \mathfrak{a}, \mathfrak{b})$ on $\mathfrak{b} \oplus \mathfrak{a}^{*}$ by

$$
\gamma=\left(\begin{array}{ll}
a & b \\
c & d
\end{array}\right): \mathfrak{b} \oplus \mathfrak{a}^{*} \rightarrow \mathfrak{b} \oplus \mathfrak{a}^{*}:(\alpha, \beta) \mapsto(\alpha, \beta) \gamma^{-1}=(d \alpha-c \beta,-b \alpha+a \beta) .
$$

A cusp of $M(\mathfrak{c}, \Gamma(N))$ is defined to be $\left(\mathfrak{a}, \mathfrak{b}, \phi_{N}\right) \bmod \mathscr{B}_{\mathbb{Q}} \cap \Gamma(N ; \mathfrak{a}, \mathfrak{b})$, where $\mathscr{B}_{\mathbb{Q}}$ is the standard Borel subgroup of $\operatorname{Res}_{\mathbb{Q}}^{F} G L_{2}$.

Let $\Sigma=\left\{\Sigma_{s}\right\}$ be a collection of $\Gamma(N)$-admissible polyhedral cone decompositions [Hida 2004, Section 4.1.4], and $\bar{M}=\bar{M}_{R}=\bar{M}_{R, N}=\bar{M}_{R, N, \Sigma}$ denotes the toroidal compactification of $M_{R}(\mathfrak{c}, \Gamma(N))$ associated to $\Sigma$. Then $\bar{M}$ is normal over $R\left[\mu_{N}\right]$. We can take $\Sigma$ such that $\bar{M}$ is smooth or projective over $R\left[\mu_{N}\right.$ ] [Hida 2004, Section 4.1.4].

Let $\widetilde{F}$ be the Galois closure of $F$ and $\mathcal{O}_{\widetilde{F}}$ the ring of integers. Then, for an $\mathcal{O}_{\widetilde{F}}\left[1 /\left(N d_{F}\right), \mu_{N}\right]$-algebra $R$, we have the isomorphism

$$
\mathrm{O}_{F} \otimes_{\mathbb{Z}} R \simeq \bigoplus_{i=1}^{g} R: a \otimes b \mapsto\left(\sigma_{1}(a) b, \sigma_{2}(a) b, \ldots, \sigma_{g}(a) b\right) .
$$

For the structure morphism $\rho: A^{U} \rightarrow M_{R}(\mathfrak{c}, \Gamma(N)), \bar{\omega}$ denotes the locally free $\mathcal{O}_{F} \otimes_{\mathbb{Z}} O_{M}$-module $\rho_{*} \Omega_{A / R}^{1}$ of rank 1. By the above isomorphism, $\bar{\omega}$ is decomposed $\bar{\omega} \simeq \bigoplus_{i} \underline{\omega}_{i}$, where $\underline{\omega}_{i}$ is the locally free $\mathcal{O}_{M}$-module of rank 1 corresponding to $\sigma_{i} \in I$. For $k=\left(k_{1}, k_{2}, \ldots, k_{g}\right) \in \mathbb{Z}^{g}$, we define the line bundle $\underline{\omega}_{R}^{k}=\underline{\omega}_{N, R}^{k}=\otimes_{i} \underline{\omega}_{i}^{\otimes k_{i}}$ called 
by the automorphic line bundle of weight $k$. When the weight $k=\left(k_{0}, k_{0}, \ldots, k_{0}\right)$ is parallel, we can construct the automorphic line bundle $\underline{\omega}_{R^{\prime}}^{k}=\left(\bigwedge^{g} \bar{\omega}\right)^{\otimes k_{0}}=\operatorname{det}(\bar{\omega})^{\otimes k_{0}}$ over every $\mathbb{Z}\left[1 /\left(N d_{F}\right), \mu_{N}\right]$-algebra $R^{\prime}$.

A c-polarized holomorphic geometric Hilbert modular form (abbreviated as GHMF) associated to $F$ of weight $k=\left(k_{1}, k_{2}, \ldots, k_{g}\right)$ and level $\Gamma(N)$ defined over $R$ is an element of $H^{0}\left(M_{R}, \underline{\omega}_{R}^{k}\right)$.

There is the semiabelian scheme $\varphi_{\text {over }} \bar{M}_{R}$ that is an extension of the universal abelian scheme $\underline{A}^{U} \rightarrow M_{R}$. Thus, $\underline{\omega}^{k}$ is extended on $\bar{M}$. When $[F: \mathbb{Q}] \geq 2$, by the Koecher principle [Chai 1990, Section 4.3], we have

$$
H^{0}\left(M_{R}, \underline{\omega}_{R}^{k}\right)=H^{0}\left(\bar{M}_{R}, \underline{\omega}_{R}^{k}\right) .
$$

Let $\rho: \mathscr{G} \rightarrow \bar{M}_{R}$ be the structure morphism and $\underline{\omega}=\operatorname{det}\left(\rho_{*} \Omega_{\mathscr{G}_{/} \bar{M}_{R}}^{1}\right)$.

By Moret-Bailly [1985, Chapter V, Theorem 2.1], $\underline{\omega}$ is semiample; i.e., there is a positive integer $n_{0}$ such that $\underline{\omega}^{\otimes n_{0}}$ is generated by global sections. Thus, the canonical rational map

$$
\phi_{\underline{\omega}^{\otimes n_{0}}}: \bar{M} \rightarrow \mathbb{P}_{R}^{r}, \quad P \mapsto\left(s_{0}(P): s_{1}(P): \cdots: s_{r}(P)\right)
$$

is a morphism, where $s_{0}, s_{1}, \ldots, s_{r} \in H^{0}\left(\bar{M}, \underline{\omega}^{\otimes n_{0}}\right)$ are global sections generating $\underline{\omega}^{\otimes n_{0}}$. We set $\pi=\pi_{N}=\phi_{\omega^{\otimes n_{0}}}$. The minimal compactification, denoted $M^{*}=M_{R}^{*}=M_{R, N}^{*}=M_{R}^{*}(\mathfrak{c}, \Gamma(N))$, of $M$ is defined to be the image of $\pi$, and

$$
M^{*} \simeq \operatorname{Proj}\left(\bigoplus_{n \geq 0} H^{0}\left(\bar{M}, \underline{\omega}^{\otimes n_{0} n}\right)\right) \text {. }
$$

By the semiampleness of $\underline{\omega}$, the graded ring $\bigoplus_{n \in \mathbb{Z}_{>0}} H^{0}\left(\bar{M}, \underline{\omega}^{\otimes n_{0} n}\right)$ is finitely generated over $R$ [Chai 1990, Section 4.4]; in particular, $M^{*}$ is of finite type over $R$. And $M$ is isomorphic to an open dense subscheme of $M^{*}$, also denoted $M$. When $g=[F: \mathbb{Q}] \geq 2$, by the Koecher principle $M^{*}$ does not depend on the choice of cone decompositions. And $M^{*}$ is also normal. The connected components $M^{*} \backslash M$ are in one-to-one correspondence with the cusps of $M$. The direct image $\pi_{*} \underline{\omega}$ is $\mathbb{Q}$-Cartier; i.e., there is a positive integer $n_{0}$ such that $\pi_{*} \underline{\omega}^{\otimes n_{0}}$ is a line bundle. Then $\pi_{*} \underline{\omega}^{\otimes n_{0}}$ is ample [Chai 1990, Section 4.3].

There is another useful definition equivalent to the above definition. We consider the covariant functor $\mathscr{P}=\mathscr{P}_{R}(\mathfrak{c}, \Gamma(N)): R$-Alg $\rightarrow$ Sets with

$$
\begin{aligned}
\mathscr{P}(B)=\left\{(\underline{A}, \omega)_{/ B} / \text { isom. } \mid \underline{A} \in \mathscr{E}(B), \rho: A \rightarrow\right. & \operatorname{Spec}(B), \\
& \left.H^{0}\left(A, \rho_{*} \Omega_{A / B}^{1}\right) \simeq\left(\mathscr{O}_{F} \otimes B\right) \omega\right\} .
\end{aligned}
$$

Then for every $R$-algebra $B, \mathscr{T}_{\mathbb{Z}}(B)=\left(\mathscr{O}_{F} \otimes_{\mathbb{Z}} B\right)^{\times}$acts on $\mathscr{P}(B)$, and $\mathscr{P} / \mathscr{T}_{\mathbb{Z}} \simeq \mathscr{E}$. If $\mathscr{E}$ is representable, so is $\mathscr{P}$. And $\mathcal{M}$ denotes the moduli scheme over $R$ representing

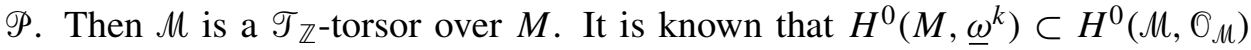
[Dimitrov and Tilouine 2004, Remarque 4.5]. Thus, $f \in H^{0}\left(M, \underline{\omega}^{k}\right)$ is regarded as 
a function such that a pair $(\underline{A}, \omega)_{/ B}$, for every $R$-algebra $B$, associates an element $f\left((\underline{A}, \omega)_{/ B}\right) \in B$ that satisfies the following:

(1) The value of $f$ depends only on the isomorphism class of $(\underline{A}, \omega)$.

(2) Given a base change map $\rho: B \rightarrow B^{\prime}, f$ satisfies

$$
\rho\left(f\left((\underline{A}, \omega)_{/ B}\right)\right)=f\left((\underline{A}, \omega) / B^{\prime}\right) .
$$

(3) For every $\alpha \in\left(\mathscr{O}_{F} \otimes_{\mathbb{Z}} B\right)^{\times}=\mathscr{T}_{\mathbb{Z}}(B)$,

$$
f(\underline{A}, \alpha \omega)=\prod_{i=1}^{g} \sigma_{i}(\alpha)^{-k_{i}} f(\underline{A}, \omega) .
$$

Let $R=\widetilde{O}_{\widetilde{F}}\left[1 /\left(N d_{F}\right), \mu_{N}\right]$, and let $B$ be an $R$-algebra and $f$ a c-polarized GHMF of weight $k=\left(k_{1}, k_{2}, \ldots, k_{g}\right)$ and level $\Gamma(N)$ defined over $B$. Let $\mathfrak{a}$ and $\mathfrak{b} \subset F$ be nonzero fractional ideals such that $\mathfrak{a} \mathfrak{b}^{-1}=\mathfrak{c}, \phi_{N}:\left(\mathfrak{b} \oplus \mathfrak{a}^{*}\right) / N\left(\mathfrak{b} \oplus \mathfrak{a}^{*}\right) \simeq\left(\widehat{O}_{F} / N \widehat{O}_{F}\right)^{2}$ an isomorphism, $s$ a cusp of $\Gamma(N)$ parametrized by $\left(\mathfrak{a}, \mathfrak{b}, \phi_{N}\right)$, and $\sigma$ a cone in the cone decomposition $\Sigma_{s}$. We set

$$
R_{\sigma}(N)=R\left[q^{\xi}\right]_{\xi \in N^{-1} \mathfrak{a} \mathfrak{b} \cap \sigma^{\vee}} \quad \text { and } \quad R_{0}(N)=R\left[q^{\xi}\right]_{\xi \in N^{-1}(\mathfrak{a} \mathfrak{b})_{+} \cup\{0\}},
$$

where $\sigma^{\vee}$ is the dual of $\sigma$. Let $S_{\sigma}(N)=\operatorname{Spec} R_{\sigma}(N)$ and $S_{0}(N)=\operatorname{Spec} R_{0}(N)$. Let $\hat{S}_{\sigma}(N)$ be the formal completion of $S_{\sigma}(N)$ along $S_{\sigma}^{\infty}(N)=S_{\sigma}(N) \backslash S_{0}(N)$. The formal scheme $\hat{S}_{\sigma}(N)$ is affine, and we define $\hat{R}_{\sigma}(N)$ as its coordinate ring. Then we can show that

$$
R \llbracket q^{\xi} \rrbracket_{\xi \in N^{-1}(\mathfrak{a b})_{+} \cup\{0\}} \hookrightarrow \hat{R}_{\sigma}(N) .
$$

The Tate object Tate $_{(\mathfrak{a}, \mathfrak{b})}(q)$ corresponding to the cusp $s$ is a Hilbert-Blumenthal abelian variety defined over the quotient field $Q\left(\hat{R}_{\sigma}(N) \otimes_{R} B\right)$. The $q$-expansion of $f$ at the cusp $s$ is defined to be the value of $f$ at the Tate object corresponding to $s$ :

$$
f\left(\text { Tate }_{(\mathfrak{a}, \mathfrak{b})}(q), \phi_{N}, \omega\right)=a_{0}+\sum_{\xi \in N^{-1} \mathfrak{a} \mathfrak{b} \cap \sigma^{\vee}} a_{\xi} q^{\xi} \in Q\left(\hat{R}_{\sigma}(N) \otimes_{R} B\right),
$$

where $\omega$ is the canonical nonvanishing differential form on Tate $_{(\mathfrak{a}, \mathfrak{b})}(q)$.

The following fact is known as the $q$-expansion principle:

Lemma 4. Let $B^{\prime}$ be a subring of $B$ such that $B^{\prime}$ is an $R$-algebra, and let $f$ be as above. Then $f$ is defined over $B^{\prime}$ if and only if $a_{\xi} \in B^{\prime}$ for all $\xi \in(\mathfrak{a} \mathfrak{b})_{+} \cup\{0\}$.

Proof. See [Rapoport 1978, Theorem 6.7].

There is a correspondence between the classical Hilbert modular forms and the geometric Hilbert modular forms defined over $\mathbb{C}$. Let $\mathfrak{H}=\mathscr{H}^{g}$ be the $g$-tuple product of the complex upper half-plane. Then the holomorphic function $f: \mathfrak{H} \rightarrow \mathbb{C}$ is a 
classical Hilbert modular form of weight $k=\left(k_{1}, \ldots, k_{g}\right)$ of level $\Gamma(N ; \mathfrak{a}, \mathfrak{b})$ if $f$ satisfies the following equation:

$$
f(\gamma z)=\prod_{\sigma_{i} \in I}\left(\sigma_{i}(c) z_{i}+\sigma_{i}(d)\right)^{k_{i}} f(z) \quad \text { for all } \gamma=\left(\begin{array}{ll}
a & b \\
c & d
\end{array}\right) \in \Gamma(N ; \mathfrak{a}, \mathfrak{b}) .
$$

Here $M_{k}(\Gamma(N ; \mathfrak{a}, \mathfrak{b}))$ denotes the complex vector space of the classical Hilbert modular forms of weight $k$ and level $\Gamma(N ; \mathfrak{a}, \mathfrak{b})$.

To show corollaries of our main result, we need the following lemma:

Lemma 5. As $\mathbb{C}$-vector spaces, $M_{k}(\Gamma(N ; \mathfrak{a}, \mathfrak{b}))$ and $H^{0}\left(\bar{M}_{\mathbb{C}}\left(\mathfrak{a} \mathfrak{b}^{-1}, \Gamma(N)\right), \underline{\omega}_{\mathbb{C}}^{k}\right)$ are canonically isomorphic.

Proof. See [Rapoport 1978, Lemme 6.12].

For $f \in M_{k}(\Gamma(N ; \mathfrak{a}, \mathfrak{b}))$, let $f^{\prime} \in H^{0}\left(\bar{M}_{\mathbb{C}}, \underline{\omega}_{\mathbb{C}}^{k}\right)$ be the form corresponding to $f$. We remark that replacing $q^{\xi}$ by $e^{2 \pi i \operatorname{Tr}(\xi z)}$, the $q$-expansion of $f^{\prime}$ at a cusp $s$ corresponds to the Fourier expansion of $f$ at the cusp $s$.

Remark 6. Let $K$ be a field that is a $\mathbb{Z}\left[1 /\left(N d_{F}\right), \mu_{N}\right]$-algebra. For the open Hilbert modular variety $M_{K} \subset \bar{M}_{K}$, the Kodaira-Spencer isomorphism [Katz 1978, Section 1.0] gives $\bar{\omega} \otimes_{\mathfrak{O}_{F} \otimes \mathbb{O}_{M}} \bar{\omega} \simeq \Omega_{M / K}^{1}$. Therefore, $\underline{\omega}_{K}^{\otimes 2}=\underline{\omega}_{K} \otimes_{\mathfrak{O}_{M}} \underline{\omega}_{K} \simeq$ $\operatorname{det}\left(\bar{\omega} \otimes_{\mathcal{O}_{F} \otimes \mathcal{O}_{M}} \bar{\omega}\right) \simeq \Omega_{M / K}^{g}$. Observing the behaviors of sections at cusps, we have

$$
\underline{\omega}^{\otimes 2} \simeq \Omega_{\bar{M} / K}^{g}\left(\log D_{\infty}\right) \simeq 0_{\bar{M}}\left(K_{\bar{M}}+D_{\infty}\right),
$$

where $K_{\bar{M}}$ is the canonical divisor of $\bar{M}$, and the Cartier divisor $D_{\infty}=\sum E$, where $E$ runs over the irreducible components of codimension 1 in $\bar{M}_{K} \backslash M_{K}$.

\section{Proofs of the main theorems}

3.1. Main result. We assume $g=[F: \mathbb{Q}] \geq 2$ so that we use intersection theory. Let $N$ be a positive integer such that $N \geq 3, k=\left(k_{1}, \ldots, k_{g}\right) \in \mathbb{Z}_{\geq 0}^{g}, \mathfrak{c}$ a nonzero integral ideal of $F$, and $K$ a field that is a $\mathbb{Z}\left[1 /\left(N d_{F}\right), \mu_{N}\right]$-algebra if $k$ is parallel or an $\mathbb{O}_{\widetilde{F}}\left[1 /\left(N d_{F}\right), \mu_{N}\right]$-algebra otherwise. Let $M_{K}=M_{K, N}=M_{K}(\mathfrak{c}, \Gamma(N))$ be the moduli scheme over $K$ defined in Section 2.2. We choose a collection of projective $\Gamma(N)$-admissible polyhedral cone decompositions $\Sigma=\left\{\Sigma_{s}\right\}$. Let $\bar{M}_{K}=\bar{M}_{K, N}=\bar{M}_{K, N, \Sigma}$ be the toroidal compactification of $M_{K}$ associated to $\Sigma$, $M_{K}^{*}=M_{K, N}^{*}$ the minimal compactification of $M_{K}$, and $\pi=\pi_{N}: \bar{M}_{K} \rightarrow M_{K}^{*}$ the canonical morphism defined in Section 2.2. We set $D_{\infty}=\sum E$, where $E$ runs over the irreducible components of codimension 1 in $\bar{M}_{K} \backslash M_{K}$. Let $S$ be a nonempty finite set of irreducible components of codimension 1 in $\bar{M}_{K} \backslash M_{K}$. For a closed point $P \in M_{K}^{*}, \mathscr{I}_{P}$ denotes the ideal sheaf on $M_{K}^{*}$ defining $P$. And $\mathfrak{I}=\mathfrak{I}_{N}$ denotes the inverse image ideal sheaf $\pi_{N}^{-1}\left(\otimes_{P \in M_{K}^{*} \backslash M_{K}} \Phi_{P}\right) \cdot \mathcal{O}_{\bar{M}_{K}}$.

For convenience, we restate Theorem 1. 
Theorem 1. Retain the notation above and let $f$ be a c-polarized geometric Hilbert modular form over $K$ of weight $k$ and level $\Gamma(N)$, i.e., $f \in H^{0}\left(\bar{M}_{K}(\Gamma(N), \mathfrak{c}), \underline{\omega}^{k}\right)$. Then, if $f \not \equiv 0$, we have $\min _{E \in S}\left\{\operatorname{ord}_{E}(f)\right\}<\kappa$ for

$$
\kappa=\kappa_{S}=\kappa_{S}(k, N)=C^{g-1} \sum_{i=1}^{g} \frac{k_{i}\left\{\left(\underline{\omega}^{(g-1)} \cdot \underline{\omega}_{i}\right)+\left(\mathfrak{I}^{(g-1)} \cdot \underline{\omega}_{i}\right)\right\}}{\left(\mathfrak{I}^{(g-1)} \cdot \sum_{E \in S} E\right)},
$$

where $C$ is a positive integer which is independent of $k$ or $N$.

Remark 6. If $f$ is a cusp form, $\kappa$ can be smaller. More generally, for a nonnegative integer $a$, if $f$ has zeros of order $a$ at all irreducible components of codimension 1 in $\bar{M} \backslash M$, we may take $\kappa$ as

$$
\kappa=C^{g-1} \sum_{i=1}^{g} \frac{k_{i}\left\{\left(\underline{\omega}^{(g-1)} \cdot \underline{\omega}_{i}\right)+\left(\mathfrak{I}^{(g-1)} \cdot \underline{\omega}_{i}\right)\right\}}{\left(\mathfrak{I}^{(g-1)} \cdot \sum_{E \in S} E\right)}-a\left(\frac{\left(\mathfrak{I}^{(g-1)} \cdot D_{\infty}\right)}{\left(\mathfrak{I}^{(g-1)} \cdot \sum_{E \in S} E\right)}-1\right) .
$$

Remark 7. When the weight is parallel, $k=\left(k_{0}, \ldots, k_{0}\right)$, we have

$$
\sum_{i} k_{0}\left(\mathfrak{I}^{(g-1)} \cdot \underline{\omega}_{i}\right)=k_{0}\left(\mathfrak{I}^{(g-1)} \cdot \underline{\omega}\right)=0
$$

by the projection formula. This implies

$$
\kappa=\frac{k_{0} C^{g-1}\left(\underline{\omega}^{(g)}\right)}{\left(\mathfrak{I}^{(g-1)} \cdot \sum_{E \in S} E\right)} .
$$

By Hirzebruch's proportionality theorem [Mumford 1977, Theorem 3.2], we have

$$
\left(\underline{\omega}^{(g)}\right)=A \operatorname{vol}(\bar{M}) c_{1}\left(\Omega_{\mathbb{C}_{\mathbb{C}}^{g}}^{1}\right)^{g}=A^{\prime}(-1)^{g} \zeta_{F}(-1)[\Gamma(1 ; \mathfrak{a}, \mathfrak{b}): \Gamma(N ; \mathfrak{a}, \mathfrak{b})],
$$

where $A$ and $A^{\prime}$ are positive constants not depending on the level $N$. In this case, we obtain

$$
\kappa=k_{0} C^{g-1} A^{\prime}(-1)^{g} \zeta_{F}(-1) \frac{[\Gamma(1 ; \mathfrak{a}, \mathfrak{b}): \Gamma(N ; \mathfrak{a}, \mathfrak{b})]}{\left(\mathfrak{I}^{(g-1)} \cdot \sum_{E \in S} E\right)} .
$$

To prove Theorem 1, we introduce the following lemma:

Lemma 8. For a sufficiently large integer $C, \underline{\omega}^{\otimes C} \otimes \mathfrak{I}$ is ample on $\bar{M}_{K}$.

Proof. We know that $\pi_{*} \underline{\omega}$ is $\mathbb{Q}$-Cartier; i.e., $\pi_{*} \underline{\omega}^{\otimes k_{0}}$ is a line bundle on $M_{K}^{*}$ for a sufficiently large $k_{0}$. Then this is ample [Chai 1990, Section 4.3]. Since $\bar{M}_{K}$ is the normalization of the blowing-up of $M_{K}^{*}$ along the ideal sheaf $\otimes_{P \in M_{K}^{*} \backslash M_{K}} \mathscr{I}_{P}$ [Ash et al. 1975, Chapter IV; Faltings and Chai 1990, Chapter V, Theorem 5.8], I is $\pi$-ample.

Lemma 2 implies the line bundle $\left(\pi^{*} \pi_{*} \underline{\omega}\right)^{\otimes k_{0} n} \otimes \mathfrak{I}$ is ample for an integer $n \gg 0$. Because $\underline{\omega}$ is semiample, $\pi^{*} \pi_{*} \underline{\omega}^{\otimes k_{0}} \simeq \underline{\omega}^{\otimes k_{0}}$. Thus, $\underline{\omega}^{\otimes k_{0} n} \otimes \mathfrak{I}$ is also ample for $n$. Replacing $k_{0} n$ with $C$, we obtain the lemma. 
Proof of Theorem 1. We assume that $f \not \equiv 0$ and set

$$
v=\min _{E \in S}\left\{\operatorname{ord}_{E}(f)\right\} .
$$

Then $f \in H^{0}\left(\bar{M}_{K}, \underline{\omega}^{k}\left(-v \sum_{E \in S} E\right)\right)$. Thus,

$$
\mathcal{O}_{\bar{M}_{K}}(\operatorname{div}(f)) \subset \underline{\omega}^{k}\left(-v \sum_{E \in S} E\right),
$$

and they are effective. By positivity of ample line bundles,

$$
\left(\left(\underline{\omega}^{\otimes C} \otimes \mathfrak{I}\right)^{(g-1)} \cdot \underline{\omega}^{k}\left(-v \sum_{E \in S} E\right)\right)>0
$$

for the integer $C$ in Lemma 8. Thus, we have

$$
v<C^{g-1} \sum_{i=1}^{g} \frac{k_{i}\left\{\left(\underline{\omega}^{(g-1)} \cdot \underline{\omega}_{i}\right)+\left(\mathfrak{I}^{(g-1)} \cdot \underline{\omega}_{i}\right)\right\}}{\left(\mathfrak{I}^{(g-1)} \cdot \sum_{E \in S} E\right)} .
$$

The independence of $C$ and $k$ and $N$ refers to Lemma 14.

Next we apply Theorem 1 for classical Hilbert modular forms. For this purpose, we start with a review of the relation between smooth cone decompositions and local structures of $\bar{M}$ at cusps.

We assume that the cone decompositions in $\Sigma$ are smooth. Let $s$ be a cusp of $M(\mathfrak{c}, \Gamma(N))$ parametrized by $\left(\mathfrak{a}, \mathfrak{b}, \phi_{N}\right)$, where $\mathfrak{a}$ and $\mathfrak{b}$ are two nonzero fractional ideals such that $\mathfrak{a b}^{-1}=\mathfrak{c}$ and $\phi_{N}: \mathfrak{b} \oplus \mathfrak{a}^{*} / N\left(\mathfrak{b} \oplus \mathfrak{a}^{*}\right) \simeq\left(\mathscr{O}_{F} / N \mathcal{O}_{F}\right)^{2}$ is an isomorphism (see Section 2.2). We can take the quotient

$$
\Sigma_{s} / U_{N}=\bigcup_{i=1}^{g}\left\{\overline{\sigma_{i, j}}\right\}_{j=1}^{r_{i}},
$$

where $U_{N}=\left\{\epsilon \in \mathcal{O}_{F}^{\times} \mid \epsilon \equiv 1 \bmod N\right\}, r_{i}$ is a positive integer, and $\overline{\sigma_{i, j}} \equiv \sigma_{i, j} \bmod U_{N}$ with an $i$-dimensional cone $\sigma_{i, j} \in \Sigma_{s}$. Let $1 \leq d \leq g$ and $\sigma \in \Sigma_{s}$ be a smooth $d$-dimensional cone. Since $\sigma$ is smooth, we can take part of a $\mathbb{Z}$-basis $\alpha_{1}, \ldots, \alpha_{d}$ of $N^{-1} \mathfrak{a} \mathfrak{b} \cap \sigma^{\vee}$ such that $N^{-1} \mathfrak{a} \mathfrak{b} \cap \sigma^{\vee}=\mathbb{Z}_{\geq 0} \alpha_{1}+\cdots+\mathbb{Z}_{\geq 0} \alpha_{d}+\mathbb{Z} \beta_{d+1}+\cdots+\mathbb{Z} \beta_{g}$ with some part of a $\mathbb{Z}$-basis $\beta_{d+1}, \ldots, \beta_{g}$ [Hida 2004, Section 4.1.4]. We regard 0 as a cone in $\Sigma_{s}$. Then $0^{\vee}=F_{\infty}=F \otimes_{\mathbb{Q}} \mathbb{R}$. We remark that $0^{\vee}=\bigcup_{\sigma \in \Sigma_{s}} \sigma^{\vee}$; in particular, $0^{\vee} \supset \sigma^{\vee}$. Thus, we have $N^{-1} \mathfrak{a b} \cap 0^{\vee}=\mathbb{Z} \alpha_{1}+\cdots+\mathbb{Z} \alpha_{d}+\mathbb{Z} \beta_{d+1}+\cdots+\mathbb{Z} \beta_{g}$ for the above $\mathbb{Z}$-basis $\alpha_{1}, \ldots, \beta_{g}$.

For the above cone $\sigma \in \Sigma_{s}$ and the ring $R=\mathbb{Z}\left[1 /\left(N d_{F}\right), \mu_{N}\right]$, the $\operatorname{ring} \hat{R}_{\sigma}(N)$ defined in Section 2.2 is the completion of $R_{\sigma}(N)$ by the principal ideal $\left(q^{\alpha_{1}} \cdots q^{\alpha_{d}}\right)$. We set

$$
\hat{R}_{\sigma}^{0}(N)=\hat{R}_{\sigma}(N) \otimes_{R_{\sigma}(N)} R_{0}(N) .
$$


It is easy to show that, if $\tau \in \Sigma_{s}$ is a face of $\sigma, \operatorname{Spec}\left(\hat{R}_{\tau}(N)\right) \subset \operatorname{Spec}\left(\hat{R}_{\sigma}(N)\right)$. The exceptional locus of $\pi: \bar{M} \rightarrow M^{*}$ is defined by gluing $\operatorname{Spec}\left(\hat{R}_{\sigma}(N)\right) \backslash \operatorname{Spec}\left(\hat{R}_{\sigma}^{0}(N)\right)$ along $\Sigma_{s} / U_{N}$ by the above rule.

For one element $\alpha_{i}$ of the basis, the divisor $E_{i}$ associated to $\alpha_{i}$ is defined by the closed irreducible subvariety of codimension 1 in $\bar{M}$ that contains the affine subvariety defined by $\left\{q^{\alpha_{i}}=0\right\}$ in the exceptional locus.

For a GHMF $f$ over a field $K$ that is an $\widetilde{O}_{\widetilde{F}}\left[1 /\left(N d_{F}\right), \mu_{N}\right]$-algebra and its $q$-expansion

$$
f=\sum_{\xi \in N^{-1}(\mathfrak{a} \mathfrak{b})_{+} \cup\{0\}} a_{\xi} q^{\xi}
$$

at the cusp $s$, the order of $f$ at $E_{i, K}=E_{i} \times \operatorname{Spec}(K)$ is

$$
\operatorname{ord}_{E_{i, K}}(f)=\min \left\{m_{i} \in \mathbb{Z} \mid a_{m_{1} \alpha_{1}+\cdots+m_{g} \alpha_{g}} \neq 0 \text { in } K\right\} .
$$

Corollary 9. Let $f \in M_{k}(\Gamma(N ; \mathfrak{a}, \mathfrak{b}))$ and

$$
f(z)=\sum_{\xi \in N^{-1}(\mathfrak{a} \mathfrak{b})_{+} \cup\{0\}} a_{\xi} e^{2 \pi i \operatorname{Tr}(\xi z)}
$$

be the Fourier expansion of $f$ at cusp s. We fix a g-dimensional cone $\sigma \in \Sigma_{s}$. Let $\left\{\alpha_{1}, \ldots, \alpha_{g}\right\}$ be a $\mathbb{Z}$-basis of $N^{-1}(\mathfrak{a} \mathfrak{b})$ corresponding to $\sigma$ and $E_{i}$ the divisor associated to $\alpha_{i}$. For a fixed $i \in\{1,2, \ldots, g\}$, we set

$$
\begin{aligned}
\kappa_{i} & =C^{g-1} \sum_{j=1}^{g} \frac{k_{j}\left\{\left(\underline{\omega}^{(g-1)} \cdot \underline{\omega}_{j}\right)+\left(\mathfrak{I}^{(g-1)} \cdot \underline{\omega}_{j}\right)\right\}}{\left(\mathfrak{I}^{(g-1)} \cdot E_{i, \mathbb{C})}\right.}, \\
\mathscr{Y}_{i} & =\left\{\xi \in m_{1} \alpha_{1}+\cdots+m_{g} \alpha_{g} \in N^{-1}(\mathfrak{a} \mathfrak{b})_{+} \cup\{0\} \mid 0 \leq m_{i} \leq \kappa_{i}\right\},
\end{aligned}
$$

where $C$ is the integer in Theorem 1. Then, if $a_{\xi}=0$ for every $\xi \in \mathscr{Y}_{i}$, we have $a_{\xi}=0$ for every $\xi \in N^{-1}(\mathfrak{a b})_{+} \cup\{0\}$.

Proof. By Lemma 5, $f$ is regarded as a global section of $H^{0}\left(\bar{M}_{\mathbb{C}}, \underline{\omega}^{k}\right)$, and the $q$-expansion of $f$ at cusp $s=\left(\mathfrak{a}, \mathfrak{b}, \phi_{N}\right)$ is

$$
f\left(\text { Tate }_{(\mathfrak{a}, \mathfrak{b})}, \phi_{N}, \omega\right)=\sum_{\xi \in N^{-1}(\mathfrak{a} \mathfrak{b})_{+} \cup\{0\}} a_{\xi} q^{\xi},
$$

where $\omega$ is a nonvanishing differential form on $\operatorname{Tate}_{(\mathfrak{a}, \mathfrak{b})}$. Applying Theorem 1 for $f$ as $K=\mathbb{C}$ and $S=\left\{E_{i, \mathbb{C}}\right\}$, the corollary is proved.

Remark 10. We can easily show that the subset $\mathscr{S}_{i}$ is a finite set. Indeed, by Proposition 13 and an easy calculation, we have an upper bound

$$
\# \mathscr{S}_{i} \ll \max \left\{k_{i}\right\}^{g} N^{3 g^{2}} .
$$

Remark 11. Corollary 9 gives a different proof of the result of [Baba et al. 2002]. 
Next we discuss the case of positive characteristic. The following corollary is an analogue of Sturm's theorem:

Corollary 12. Let $f \in M_{k}(\Gamma(N ; \mathfrak{a}, \mathfrak{b}))$ and

$$
f(z)=\sum_{\xi \in N^{-1}(\mathfrak{a b})_{+} \cup\{0\}} a_{\xi} e^{2 \pi i \operatorname{Tr}(\xi z)}
$$

be the Fourier expansion of $f$ at the cusp $s=\left(\mathfrak{a}, \mathfrak{b}, \phi_{N}\right)$. We assume $g=[F: \mathbb{Q}] \geq 2$ and fix a g-dimensional cone $\sigma \in \Sigma_{s}$. Let $\left\{\alpha_{1}, \ldots, \alpha_{g}\right\}$ be a $\mathbb{Z}$-basis of $N^{-1}(\mathfrak{a} \mathfrak{b})$ corresponding to $\sigma$ and $E_{i}$ the divisor associated to $\alpha_{i}$. Let $L$ be a number field, $\mathscr{O}_{L}$ the ring of integers of $L$, and $\lambda$ a prime ideal in $\mathrm{O}_{L}$ such that $\lambda \nmid N d_{F} \mathrm{O}_{L}$. Assume $a_{\xi} \in \mathrm{O}_{L}$ for every $\xi$. For a fixed $i \in\{1,2, \ldots, g\}$, we set

$$
\kappa_{i}=C^{g-1} \sum_{j=1}^{g} \frac{k_{j}\left\{\left(\underline{\omega}^{(g-1)} \cdot \underline{\omega}_{j}\right)+\left(\mathfrak{I}^{(g-1)} \cdot \underline{\omega}_{j}\right)\right\}}{\left(\mathfrak{I}^{(g-1)} \cdot E_{i, \mathbb{E}}\right)},
$$

where $C$ is the integer in Theorem 1 and $\mathbb{F}$ is a finite extension of $\mathrm{O}_{L} / \lambda$ (defined in the proof), and set $\mathscr{Y}_{i}$ to be same as in Corollary 9.

Then, if $a_{\xi} \equiv 0 \bmod \lambda$ for every $\xi \in \mathscr{Y}_{i}$, we have $a_{\xi} \equiv 0 \bmod \lambda$ for every $\xi$ in $N^{-1}(\mathfrak{a} \mathfrak{b})_{+} \cup\{0\}$.

Proof. Lemma 5 implies that $f$ is regarded as an element of $H^{0}\left(\bar{M}_{\mathbb{C}}, \underline{\omega}^{k}\right)$. Let $\widetilde{L}$ be the composition field of $L$ and $\widetilde{F}$. By the assumption, all the Fourier coefficients of $f$ are in $\mathcal{O}_{L}$ and thus in $\mathcal{O}_{\tilde{L}}\left[1 /\left(N d_{F}\right), \mu_{N}\right]$. Let $\lambda^{\prime}$ be a maximal ideal of ring $\mathbb{O}_{\widetilde{L}}\left[1 /\left(N d_{F}\right), \mu_{N}\right]$ such that $\lambda^{\prime} \mid \lambda \widetilde{O}_{\tilde{L}}\left[1 /\left(N d_{F}\right), \mu_{N}\right]$. Remark that $\lambda^{\prime} \cap \mathscr{O}_{L}=\lambda$. By Lemma 4 and the commutativity with base change maps of GHMF (see Section 2.2), we regard $f$ as a GHMF defined over the field $\widetilde{O}_{\widetilde{L}}\left[1 /\left(N d_{F}\right), \mu_{N}\right] / \lambda^{\prime}$, and the $q$ expansion of $f$ at $\operatorname{cusp} s=\left(\mathfrak{a}, \mathfrak{b}, \phi_{N}\right)$ is

$$
f\left(\operatorname{Tate}_{(\mathfrak{a}, \mathfrak{b})}, \phi_{N}, \omega_{\mathrm{can}}\right)=\sum_{\xi \in N^{-1}(\mathfrak{a b})_{+} \cup\{0\}}\left(a_{\xi} \bmod \lambda^{\prime}\right) q^{\xi} .
$$

Applying Theorem 1 for $f$ as $K=\mathbb{F}=\mathcal{O}_{\widetilde{L}}\left[1 /\left(N d_{F}\right), \mu_{N}\right] / \lambda^{\prime}$ and $S=\left\{E_{i, \mathbb{F}}\right\}$, if $f \not \equiv 0 \bmod \lambda^{\prime}$, an integer $m_{i}$ such that $0 \leq m_{i} \leq \kappa_{i}$ and $a_{m_{1} \alpha_{1}+\cdots+m_{g} \alpha_{g}} \not \equiv 0 \bmod \lambda^{\prime}$ exists. Thus, $a_{m_{1} \alpha_{1}+\cdots+m_{g} \alpha_{g}} \notin \lambda^{\prime} \cap \mathcal{O}_{K}=\lambda$, and the contrapositive of the corollary is proved.

Next we examine the growth of $\kappa=\kappa(k, N)$ associated to weight $k=\left(k_{1}, \ldots, k_{g}\right)$ and level $N$.

Proposition 13. We may take $\kappa$ in Theorem 1 as

$$
\kappa(k, N) \ll \max _{i}\left\{k_{i}\right\} N^{3 g} .
$$

Let $\underline{\omega}_{N}$ be the automorphic line bundle on $\bar{M}_{N}$. To show Proposition 13, we introduce the following lemma: 
Lemma 14. Let $\ell$ be the characteristic of $K, N$ and $N^{\prime}$ two positive integers such that $N \geq 3$ and $N \mid N^{\prime}$ and $\ell \nmid N^{\prime}$, and $C$ a positive integer. Then $\underline{\omega}_{N}^{\otimes C} \otimes \mathfrak{I}_{N}$ is ample if and only if $\underline{\omega}_{N^{\prime}}^{\otimes C} \otimes \mathfrak{I}_{N^{\prime}}$ is ample.

Proof. We can take a finite étale morphism $h: M_{N^{\prime}, K} \rightarrow M_{N, K}$ over $\mathbb{Z}\left[1 /\left(N^{\prime}\right)\right]$ [Chai 1990, Section 2.2]. Let $\Sigma$ be a collection of cone decompositions of level $N$ and $\bar{M}_{N, K, \Sigma}$ the toroidal compactification associated to $\Sigma$. We define $\bar{M}_{N^{\prime}, K}$ to be the normalization of $\bar{M}_{N, K}$ in $M_{N^{\prime}, K}$, and $\tilde{h}: \bar{M}_{N^{\prime}, K} \rightarrow \bar{M}_{N, K}$ denotes the normalization morphism. Then the normalization $\bar{M}_{N^{\prime}, K}$ is the toroidal compactification of $M_{N^{\prime}, K}$ associated to $\Sigma$ [Faltings and Chai 1990, Chapter IV, Theorem 6.7(1)]. Here $\Sigma$ is regarded as a collection of cone decompositions of level $N^{\prime}$. Remark that $\bar{M}_{N^{\prime}, K}$ may not be smooth. Then $\tilde{h}$ is an extension of $h$ to toroidal compactifications [Faltings and Chai 1990, Chapter IV, Theorem 6.7(2)]. In particular, $\left.\tilde{h}\right|_{M_{N^{\prime}, K}}$ is étale. We can show that $\tilde{h}^{*} \underline{\omega}_{N}^{\otimes 2} \simeq \underline{\omega}_{N^{\prime}}^{\otimes 2}$. Indeed by Remark 6 ,

$$
\underline{\omega}_{N}^{\otimes 2} \simeq \mathcal{O}_{\bar{M}_{N, K}}\left(K_{\bar{M}_{N, K}}+D_{\infty, N}\right),
$$

where $D_{\infty, N}=\sum E$ such that $E$ runs over the irreducible components of codimension 1 in $\bar{M}_{N, K} \backslash M_{N, K}$. Since $\left.\tilde{h}\right|_{M_{N^{\prime}, K}}$ is étale, the ramification of $\tilde{h}$ occurs only at $\bar{M}_{N, K} \backslash M_{N, K}$. Thus, we can show that

$$
\tilde{h}^{*} \underline{\omega}_{N}^{\otimes 2} \simeq \tilde{h}^{*} \mathcal{O}_{\bar{M}_{N, K}}\left(K_{\bar{M}_{N, K}}+D_{\infty, N}\right) \simeq \mathcal{O}_{\bar{M}_{N^{\prime}, K}}\left(K_{\bar{M}_{N^{\prime}, K}}+D_{\infty, N^{\prime}}\right) \simeq \underline{\omega}_{N^{\prime}}^{\otimes 2} .
$$

It is known that $\tilde{h}^{*} \mathfrak{I}_{N} \simeq \mathfrak{I}_{N^{\prime}}$ [Faltings and Chai 1990, Chapter V, proof of Corollary 5.14]. Assume that $C$ is even and that $\underline{\omega}_{N}^{\otimes C} \otimes \mathfrak{I}_{N}$ is ample. Since $\tilde{h}$ is finite and surjective, $\underline{\omega}_{N}^{\otimes C} \otimes \mathfrak{I}_{N}$ is ample if and only if $\underline{\omega}_{N^{\prime}}^{\otimes C} \otimes \mathfrak{I}_{N^{\prime}}$ is ample by Lemma 3 .

Proof of Proposition 13. Let $N$ and $N^{\prime}$ be positive integers such that $N, N^{\prime} \geq 3$ and $\operatorname{char}(K) \nmid N N^{\prime}$ and $C$ a positive integer such that $\underline{\omega}_{N}^{\otimes C} \otimes \mathfrak{I}_{N}$ is ample. According to Lemma $14, \underline{\omega}_{N N^{\prime}}^{\otimes C} \otimes \mathfrak{I}_{N N^{\prime}}$ is ample. Thus, $\underline{\omega}_{N^{\prime}}^{\otimes C} \otimes \mathfrak{I}_{N^{\prime}}$ is also ample. This implies that we may take $C$ independent of $N$. By Remark 7, we have

$$
\begin{aligned}
\kappa(k, N) & =C^{g-1} \sum_{i=1}^{g} \frac{k_{i}\left\{\left(\underline{\omega}^{(g-1)} \cdot \underline{\omega}_{i}\right)+\left(\mathfrak{I}^{(g-1)} \cdot \underline{\omega}_{i}\right)\right\}}{\left(\mathfrak{I}_{N}^{(g-1)} \cdot \sum_{E \in S} E\right)} \\
& \leq C^{g-1} \max _{i}\left\{k_{i}\right\}\left(\underline{\omega}^{(g)}\right) \\
& =C^{(g-1)} A \max _{i}\left\{k_{i}\right\} \zeta_{F}(-1)[\Gamma(1 ; \mathfrak{a}, \mathfrak{b}): \Gamma(N ; \mathfrak{a}, \mathfrak{b})] \\
& \ll \max _{i}\left\{k_{i}\right\} N^{3 g} .
\end{aligned}
$$

As a consequence of Theorem 1, Proposition 13, and Remark 10, we obtain an upper bound for the dimension of the vector space of the Hilbert modular forms. 
Corollary 15. Let $N$ be an integer such that $N \geq 3, k=\left(k_{1}, \ldots, k_{g}\right) \in \mathbb{Z}_{\geq 0}^{g}$ a weight vector, and $K$ a field that is an $\mathcal{O}_{\tilde{F}}\left[1 /\left(N d_{F}\right), \mu_{N}\right]$-algebra $\left(a \mathbb{Z}\left[1 /\left(\bar{N} d_{F}\right), \mu_{N}\right]\right.$ algebra if $k$ is parallel). Then

$$
\operatorname{dim}_{K} H^{0}\left(\bar{M}_{K}, \underline{\omega}^{k}\right) \ll\left(\max _{i}\left\{k_{i}\right\}\right)^{g} N^{3 g^{2}} .
$$

3.2. The case that $F$ is a real quadratic field. In this section, for some special situations, we investigate the strange constant $C$ appearing in Theorem 1.

Assume that $g=2$ and the canonical bundle $K_{\bar{M}_{K}}$ is nef. For the invertible sheaf $\mathfrak{I}_{N}$ in Theorem 1 , we set

$$
\mathrm{O}_{\bar{M}_{K}}\left(-\sum_{i} n_{i} E_{i}\right)=\mathfrak{I}_{N}
$$

where $\left\{E_{i}\right\}$ are the exceptional curves of $\pi: \bar{M}_{K} \rightarrow M_{K}^{*}$ and $n_{i}$ are positive integers. We set $n_{\max }=\max _{i}\left\{n_{i}\right\}$. Then we may take $2 n_{\max }$ as the constant $C$.

Theorem 16. In the above setting, we may take $C=2 n_{\max }$. Thus, in this situation, we may take

$$
\kappa=2 n_{\max } \frac{\sum_{j=1}^{2} k_{j}\left\{\left(\underline{\omega} \cdot \underline{\omega}_{j}\right)-\left(\sum_{i} n_{i} E_{i} \cdot \underline{\omega}_{j}\right)\right\}}{-\left(\sum_{i} n_{i} E_{i} \cdot \sum_{E \in S} E\right)} .
$$

Proof. For our purpose, we need a line bundle $\mathscr{L}$ on $\bar{M}$ that is nef and $(\mathscr{L} \cdot E)>0$ for all exceptional curves $E$. Thus, it is sufficient to prove that $\left(\omega^{\otimes 2 n_{\max }} \otimes \mathfrak{I}_{N} \cdot \mathscr{C}\right) \geq 0$ for every irreducible curve $\mathscr{C}$ in $\bar{M}_{K}$. In particular, it is not necessary to prove that the line bundle is ample. First, assume that the curve $\mathscr{C}$ is exceptional. Then, by the $\pi$-ampleness of $\mathfrak{I}_{N}$, we have

$$
\begin{aligned}
\left(\omega^{\otimes 2 n_{\max }} \otimes \mathfrak{I}_{N} \cdot \mathscr{C}\right) & =\left(\pi^{*} \pi_{*} \omega^{\otimes 2 n_{\max }} \cdot \mathscr{C}\right)+\left(\mathfrak{I}_{N} \cdot \mathscr{C}\right) \\
& =\left(\pi_{*} \omega^{\otimes 2 n_{\max }} \cdot \pi_{*} \mathscr{C}\right)+\left(\mathfrak{I}_{N} \cdot \mathscr{C}\right)=\left(\mathfrak{I}_{N} \cdot \mathscr{C}\right)>0 .
\end{aligned}
$$

Next assume $\mathscr{b}$ is an irreducible curve such that $\pi(\mathscr{b})$ is an irreducible curve. Then

$$
\begin{aligned}
& \left(\omega^{\otimes 2 n_{\max }} \otimes \mathfrak{I}_{N} \cdot \mathscr{C}\right)=\left(\mathcal{O}_{\bar{M}_{K}}\left(n_{\max } K_{\bar{M}_{K}}+n_{\max } D_{\infty}-\sum_{i} n_{i} E_{i}\right) \cdot \mathscr{C}\right) \\
& =\left(n_{\max } K_{\bar{M}_{K}} \cdot \mathscr{C}\right)+\left(\sum_{i}\left(n_{\max }-n_{i}\right) E_{i} \cdot \mathscr{b}\right) \geq 0 .
\end{aligned}
$$

We give an example for Theorem 16 in the setting of [Dieulefait et al. 2010, Appendix B].

Example 17. Let $F=\mathbb{Q}(\sqrt{5}), N=3$, and $k_{1}=k_{2}=2 k \in 2 \mathbb{Z}_{>0}$. Then there are the exceptional curves $E_{i}(i=1,2, \ldots, 10)$ and the curve $F_{1}$ defined in [van der 
Geer 1988, page 88]. These intersection numbers are as follows:

$$
\begin{aligned}
& \left(E_{i} \cdot E_{j}\right)=\left\{\begin{aligned}
0 & \text { if } i \neq j, \\
-4 & \text { if } i=j,
\end{aligned}\right. \\
& \left(F_{1} \cdot F_{1}\right)=-60, \\
& \left(E_{i} \cdot F_{1}\right)=12 \text { for } i=1,2, \ldots, 10 .
\end{aligned}
$$

We set

$$
D^{\prime}=\frac{1}{5}\left(\sum_{i=1}^{10} E_{i}+2 F_{1}\right),
$$

then $D^{\prime}$ is nef and $\left(D^{\prime} \cdot D^{\prime}\right)=8$, and it is known that $D^{\prime}$ equals the canonical divisor $K_{\bar{M}}$. In particular, $\bar{M}$ is a minimal surface of general type. Then we may take 1 as $n_{\max }$ because we may use $\mathcal{O}\left(-D_{\infty}\right)$ as the ideal sheaf $\mathfrak{I}_{3}$, where $D_{\infty}=\sum_{i=1}^{10} E_{i}$. Thus, by easy calculation, we have $\kappa=12 k$. This estimate is the same as [Dieulefait et al. 2010, Theorem B.3].

Next we consider a more particular case. Assume that the toroidal compactification $\bar{M}_{\mathbb{F}}$ is a minimal surface of general type (i.e., the canonical divisor $K_{\bar{M}_{\mathbb{F}}}$ of $\bar{M}_{\mathbb{F}}$ is nef and $\left.\left(K_{\bar{M}_{\mathbb{F}}} \cdot K_{\bar{M}_{\mathbb{F}}}\right)>0\right)$ over a field $\mathbb{F}$ of positive characteristic $\ell$. Under the above assumption, we have:

Lemma 18 [Ekedahl 1988, Chapter III, Proposition 1.13]. Let $\mathbb{F}$ be a field of positive characteristic, $X$ a minimal surface of general type over $\mathbb{F}$, and $Z$ the fundamental cycle on $X$. Then $2 K_{X}-Z$ is numerically positive.

Here the fundamental cycle of $X$ is the fundamental cycle associated to the canonical morphism $\phi=\phi_{m K_{X}}: X \rightarrow X_{\text {can }}$ with a sufficiently large integer $m$, where $X_{\text {can }}$ is the canonical model of $X$. The Weil divisor $Z$ is the fundamental cycle of $\phi$ if $Z$ is the smallest element in the set

$$
\left\{D=\sum m_{i} E_{i} \mid E_{i} \in \operatorname{Ex}(\phi), m_{i}>0,(D \cdot E) \leq 0 \text { for all } E \in \operatorname{Ex}(\phi)\right\},
$$

where $\operatorname{Ex}(\phi)$ is the set of the exceptional curves of $\phi$ (i.e., the irreducible curves contracted by $\phi$ ) on $X$. For an irreducible curve $\mathscr{C} \subset X, \mathscr{C}$ is contracted by $\phi$ if and only if $\left(K_{X} \cdot \mathscr{C}\right)=0$. If $\mathscr{C} \simeq \mathbb{P}^{1}$ and $\mathscr{C}$ is contracted by $\phi$, by the adjunction formula

$$
p_{a}(\mathscr{C})=1+\frac{1}{2}\left((\mathscr{b} \cdot \mathscr{C})+\left(K_{X} \cdot \mathscr{C}\right)\right),
$$

we have $(\mathscr{b} \cdot \mathscr{C})=-2$. Thus, $X_{\text {can }}$ is obtained by contracting all $(-2)$-curves on $X$.

Return to our case. Let $\pi$ be the morphism $\bar{M}_{\mathbb{F}} \rightarrow M_{\mathbb{F}}^{*}, \phi=\phi_{m K_{\bar{M}_{\mathbb{F}}}}: \bar{M}_{\mathbb{F}} \rightarrow \bar{M}_{\mathbb{F} \text {,can }}$ with a sufficiently large integer $m$, and $\operatorname{Ex}(\pi)$ and $\operatorname{Ex}(\phi)$ the sets of the exceptional curves of $\pi$ and $\phi$, respectively. Then we have

$$
\operatorname{Ex}(\phi)=\{E \in \operatorname{Ex}(\pi) \mid(E \cdot E)=-2\} .
$$


We can take $Z=\sum_{E \in \operatorname{Ex}(\phi)} E$ as the fundamental cycle of $\phi$. Remark 6 implies

$$
\mathcal{O}_{\bar{M}_{\mathbb{F}}}\left(2 K_{\bar{M}_{\mathbb{F}}}-Z\right) \simeq \underline{\omega}^{\otimes 4} \otimes \mathcal{O}_{\bar{M}_{\mathbb{F}}}\left(-2 D_{\infty}-Z\right) .
$$

Thus, we obtain:

Theorem 19. Let $N \geq 3$ be an integer, $k=\left(k_{1}, k_{2}\right) \in \mathbb{Z}_{\geq 0}^{2}$, $\ell$ an odd prime number such that $\ell \nmid N d_{F}, \mathfrak{c}$ a nonzero integral ideal of $F$, and $\mathbb{F}$ a field of characteristic $\ell$. Assume that $K_{\bar{M}_{\mathbb{F}}}$ is nef and $\left(K_{\bar{M}_{\mathbb{F}}} \cdot K_{\bar{M}_{\mathbb{F}}}\right)>0$. Let $S$ be nonempty finite set of exceptional curves of $\pi: \bar{M}_{\mathbb{F}} \rightarrow M_{\mathbb{F}}^{*}$. Let $f$ be a c-polarized geometric Hilbert modular form over $\mathbb{F}$ of weight $\left(k_{1}, k_{2}\right)$ and level $\Gamma(N)$. Then, if $f \neq \equiv 0$, we have $\min _{E \in S}\left\{\operatorname{ord}_{E}(f)\right\}<\kappa$. Here

$$
\kappa=\sum_{i=1}^{2} \frac{k_{i}\left\{4\left(\underline{\omega} \cdot \underline{\omega}_{i}\right)-\left(\left(2 D_{\infty}+Z\right) \cdot \underline{\omega}_{j}\right)\right\}}{\left(\left(-2 D_{\infty}-Z\right) \cdot \sum_{E \in S} E\right)},
$$

where $D_{\infty}$ and $Z$ are the above Weil divisors.

Remark 20. In the settings of Theorem $19,2 n_{\max }=6$ if $\operatorname{Ex}(\phi)$ is nonempty. Thus, when the weight is parallel $(k, k)$ and $\operatorname{Ex}(\phi)$ is not empty, Theorem 16 implies

$$
\kappa=\frac{6 k(\underline{\omega} \cdot \underline{\omega})}{\left(\left(-2 D_{\infty}-Z\right) \cdot \sum_{E \in S} E\right)} .
$$

But on the same assumptions, Theorem 19 implies

$$
\kappa=\frac{4 k(\underline{\omega} \cdot \underline{\omega})}{\left(\left(-2 D_{\infty}-Z\right) \cdot \sum_{E \in S} E\right)} .
$$

Therefore, the estimate of $\kappa$ in Theorem 19 is slightly better than in Theorem 16.

Remark 21. In the situation of Theorems 16 and 19, if $f$ is a cusp form, we can take a smaller bound as Remark 6. For example, under the assumption of Theorem 16, for $f$ having zeros of order $a$ at all the irreducible components of codimension 1 in $\bar{M} \backslash M$, the bound can be taken as

$$
\kappa-a\left(\frac{\left(\sum_{i} n_{i} E_{i} \cdot D_{\infty}\right)}{\left(\sum_{i} n_{i} E_{i} \cdot \sum_{E \in S} E\right)}-1\right) .
$$

\section{Acknowledgements}

This article is a revised version of my $\mathrm{PhD}$ thesis [Takai 2010]. I would like to thank my adviser Kazuhiro Fujiwara for his thorough guidance and invaluable discussion and Lars Hesselholt for his helpful comments concerning an earlier draft of this paper. I also gratefully acknowledge the comments by the referee. 


\section{References}

[Ash et al. 1975] A. Ash, D. Mumford, M. Rapoport, and Y. Tai, Smooth compactification of locally symmetric varieties, Lie Groups: History, Frontiers and Applications 4, Math. Sci. Press, Brookline, MA, 1975. MR 56 \#15642 Zbl 0334.14007

[Baba et al. 2002] S. Baba, K. Chakraborty, and Y. N. Petridis, "On the number of Fourier coefficients that determine a Hilbert modular form", Proc. Amer. Math. Soc. 130:9 (2002), 2497-2502. MR 2003c:11037 Zbl 1125.11323

[Chai 1990] C.-L. Chai, "Arithmetic minimal compactification of the Hilbert-Blumenthal moduli spaces”, Ann. of Math. (2) 131:3 (1990), 541-554. MR 91i:11063 Zbl 0754.14030

[Deligne and Rapoport 1973] P. Deligne and M. Rapoport, "Les schémas de modules de courbes elliptiques", pp. 143-316 in Modular functions of one variable (Antwerp, 1972), vol. 2, edited by P. Deligne and W. Kuyk, Lecture Notes in Mathematics 349, Springer, Berlin, 1973. MR 49 \#2762 Zbl 0281.14010

[Dieulefait et al. 2010] L. Dieulefait, A. Pacetti, and M. Schuett, "Modularity of the Consani-Scholten quintic", preprint, 2010. arXiv 1005.4523

[Dimitrov 2004] M. Dimitrov, "Compactifications arithmétiques des variétés de Hilbert et formes modulaires de Hilbert pour $\Gamma_{1}(\mathfrak{c}, \mathfrak{n})$ ”, pp. 527-554 in Geometric aspects of Dwork theory, edited by A. Adolphson et al., de Gruyter, Berlin, 2004. MR 2006e:11063 Zbl 1076.14029

[Dimitrov and Tilouine 2004] M. Dimitrov and J. Tilouine, "Variétés et formes modulaires de Hilbert arithmétiques pour $\Gamma_{1}(\mathfrak{c}, \mathfrak{n})$ ", pp. 555-614 in Geometric aspects of Dwork theory, edited by A. Adolphson et al., de Gruyter, Berlin, 2004. MR 2006e:11064 Zbl 1127.11036

[Doi and Ohta 1977] K. Doi and M. Ohta, "On some congruences between cusp forms on $\Gamma_{0}(N)$ ", pp. 91-105 in Modular functions of one variable (Bonn, 1976), vol. 5, Lecture Notes in Mathematics 601, Springer, Berlin, 1977. MR 57 \#235 Zbl 0361.10023

[Ekedahl 1988] T. Ekedahl, "Canonical models of surfaces of general type in positive characteristic", Inst. Hautes Études Sci. Publ. Math. 67 (1988), 97-144. MR 89k:14069 Zbl 0674.14028

[Faltings and Chai 1990] G. Faltings and C.-L. Chai, Degeneration of abelian varieties, Ergeb. Math. Grenzgeb. (3) 22, Springer, Berlin, 1990. MR 92d:14036 Zbl 0744.14031

[Fulton 1998] W. Fulton, Intersection theory, 2nd ed., Ergeb. Math. Grenzgeb. (3) 2, Springer, Berlin, 1998. MR 99d:14003 Zbl 0885.14002

[van der Geer 1988] G. van der Geer, Hilbert modular surfaces, Ergeb. Math. Grenzgeb. (3) 16, Springer, Berlin, 1988. MR 89c:11073 Zbl 0634.14022

[Hartshorne 1977] R. Hartshorne, Algebraic geometry, Graduate Texts in Mathematics 52, Springer, New York, 1977. MR 57 \#3116 Zbl 0367.14001

[Hida 2004] H. Hida, p-adic automorphic forms on Shimura varieties, Springer, New York, 2004. MR 2005e:11054 Zbl 1055.11032

[Katz 1978] N. M. Katz, “p-adic L-functions for CM fields”, Invent. Math. 49:3 (1978), 199-297. MR 80h:10039 Zbl 0417.12003

[Lazarsfeld 2004] R. Lazarsfeld, Positivity in algebraic geometry, I: Classical setting, line bundles and linear series, Ergeb. Math. Grenzgeb. (3) 48, Springer, Berlin, 2004. MR 2005k:14001a Zbl 1093.14501

[Miyake 1989] T. Miyake, Modular forms, Springer, Berlin, 1989. MR 90m:11062 Zbl 0701.11014 [Moret-Bailly 1985] L. Moret-Bailly, Pinceaux de variétés abéliennes, Astérisque 129, Société Mathématique de France, Paris, 1985. MR 87j:14069 Zbl 0595.14032 
[Mumford 1977] D. Mumford, "Hirzebruch's proportionality theorem in the noncompact case", Invent. Math. 42 (1977), 239-272. MR 81a:32026 Zbl 0365.14012

[Rapoport 1978] M. Rapoport, "Compactifications de l'espace de modules de Hilbert-Blumenthal", Compositio Math. 36:3 (1978), 255-335. MR 80j:14009 Zbl 0386.14006

[Sturm 1987] J. Sturm, "On the congruence of modular forms", pp. 275-280 in Number theory (New York, 1984-1985), edited by D. V. Chudnovsky et al., Lecture Notes in Mathematics 1240, Springer, Berlin, 1987. MR 88h:11031 Zbl 0615.10035

[Takai 2010] Y. Takai, An analogy of Sturm's theorem for real quadratic fields, Ph.D. thesis, Nagoya University, 2010.

Communicated by Richard Taylor

Received 2011-11-22 Revised 2012-08-30 Accepted 2012-09-04

takai@ms.u-tokyo.ac.jp Graduate School of Mathematical Sciences, University of Tokyo, 3-8-1 Komaba, Meguro, Tokyo, 153-8914, Japan 


\section{Algebra \& Number Theory}

msp.org/ant

\section{EDITORS}

MANAGING EDITOR

Bjorn Poonen

Massachusetts Institute of Technology

Cambridge, USA

\author{
EDITORIAL BOARD CHAIR \\ David Eisenbud \\ University of California \\ Berkeley, USA
}

\section{BOARD OF EDITORS}

Georgia Benkart

Dave Benson

Richard E. Borcherds

John H. Coates

J-L. Colliot-Thélène

Brian D. Conrad

Hélène Esnault

Hubert Flenner

Edward Frenkel

Andrew Granville

Joseph Gubeladze

Ehud Hrushovski

Craig Huneke

Mikhail Kapranov

Yujiro Kawamata

János Kollár

Yuri Manin

Barry Mazur

Philippe Michel
University of Wisconsin, Madison, USA

University of Aberdeen, Scotland

University of California, Berkeley, USA

University of Cambridge, UK

CNRS, Université Paris-Sud, France

University of Michigan, USA

Freie Universität Berlin, Germany

Ruhr-Universität, Germany

University of California, Berkeley, USA

Université de Montréal, Canada

San Francisco State University, USA

Hebrew University, Israel

University of Virginia, USA

Yale University, USA

University of Tokyo, Japan

Princeton University, USA

Northwestern University, USA

Harvard University, USA

École Polytechnique Fédérale de Lausanne
Susan Montgomery

Shigefumi Mori

Raman Parimala

Jonathan Pila

Victor Reiner

Karl Rubin

Peter Sarnak

Joseph H. Silverman

Michael Singer

Vasudevan Srinivas

J. Toby Stafford

Bernd Sturmfels

Richard Taylor

Ravi Vakil

Michel van den Bergh

Marie-France Vignéras

Kei-Ichi Watanabe

Efim Zelmanov
University of Southern California, USA

RIMS, Kyoto University, Japan

Emory University, USA

University of Oxford, UK

University of Minnesota, USA

University of California, Irvine, USA

Princeton University, USA

Brown University, USA

North Carolina State University, USA

Tata Inst. of Fund. Research, India

University of Michigan, USA

University of California, Berkeley, USA

Harvard University, USA

Stanford University, USA

Hasselt University, Belgium

Université Paris VII, France

Nihon University, Japan

University of California, San Diego, USA

\section{PRODUCTION}

production@msp.org

Silvio Levy, Scientific Editor

See inside back cover or msp.org/ant for submission instructions.

The subscription price for 2013 is US \$200/year for the electronic version, and \$350/year ( $\$ 40$, if shipping outside the US) for print and electronic. Subscriptions, requests for back issues and changes of subscribers address should be sent to MSP.

Algebra \& Number Theory (ISSN 1944-7833 electronic, 1937-0652 printed) at Mathematical Sciences Publishers, 798 Evans Hall \#3840, c/o University of California, Berkeley, CA 94720-3840 is published continuously online. Periodical rate postage paid at Berkeley, CA 94704, and additional mailing offices.

ANT peer review and production are managed by EditFLOW ${ }^{\circledR}$ from Mathematical Sciences Publishers.

\section{PUBLISHED BY}

- mathematical sciences publishers

nonprofit scientific publishing

http://msp.org/

(C) 2013 Mathematical Sciences Publishers 


\section{Algebra \& Number Theory}

$\begin{array}{lll}\text { Volume } 7 & \text { No. } 4 \quad 2013\end{array}$

Explicit Chabauty over number fields

SAMIR SIKSEK

Moduli spaces for point modules on naïve blowups

Thomas A. Nevins and Susan J. Sierra

Density of rational points on certain surfaces

Sir Peter SWInNerton-Dyer

HENRIK RUSSELL

Chai's conjecture and Fubini properties of dimensional motivic integration

RAF Cluckers, François LoESER and JohanNes NiCAISE

SHUNSUKE TAKAGI

Finitely presented exponential fields

JONATHAN KIRBY

On a problem of Arnold: The average multiplicative order of a given integer

PÄr Kurlberg and CARL POMERANCE

An analogue of Sturm's theorem for Hilbert modular forms 